\title{
CAPÍTULO XIII
}

\section{Generación de residuos sólidos urbanos: determinantes para el caso colombiano}

\author{
María del Pilar Sánchez Muñoz \\ María Gabriela Ramos Barrera**
}

* Candidata a Doctora en Desarrollo Sostenible, Magíster en Economía, Economista. Docente de la Facultad de Ciencias Contables, Económicas y Administrativas de la Universidad de Manizales y de la Escuela de Administración de la Universidad del Rosario. Investigadora del Grupo Economía y Desarrollo Humano de la Universidad de La Salle, con investigaciones publicadas en revistas indexadas, nacionales e internacionales.

** Magíster en Economía Cuantitativa, Economista. Docente del Programa de Negocios Internacionales de la Facultad de Negocios, Gestión y Sostenibilidad de la Institución Universitaria Politécnico Grancolombiano, con investigaciones publicadas en revistas indexadas, nacionales e internacionales. 


\section{Introducción}

Desde la década de los setenta la preocupación que se ha puesto de manifiesto por diferentes organizaciones internacionales ha sido el efecto negativo de las actividades antrópicas sobre los recursos naturales que se están agotando, comprometiendo la vida de las generaciones futuras. En 1987 la Asamblea General de las Naciones Unidas, haciendo evidente esta problemática, preparó un informe denominado "Nuestro Futuro Común", más conocido como el Informe Brundtland, a partir del cual se vienen proponiendo alternativas para garantizar un desarrollo sostenible con la producción de bienes y servicios para la satisfacción de necesidades, el uso adecuado de los servicios ambientales, la eficiencia energética en el uso de tecnologías y el progreso en la forma como los individuos interactúan con su entorno. Considerando que en los diferentes procesos del ciclo económico, no sólo se transforman recursos naturales en bienes y servicios para el consumo final, sino que también se generan una gran cantidad de residuos sólidos (RS), su manejo y disposición se debe realizar de una forma eficiente; además, la capacidad de los ecosistemas para absorber dichos residuos no es suficiente comparada con la generación de los mismos.

Lo anterior, hace imperativo que los desechos generados vuelvan al ciclo económico como materias primas, que la disposición seleccionada genere la menor cantidad de vectores y gas, y que los actores involucrados en su manejo, como es el caso de los recicladores, reciban por su trabajo un ingreso que dignifique la labor. Actividades como la producción verde, el consumo responsable, la separación en fuente de los residuos; son indispensables para alcanzar el desarrollo sostenible en la medida en que respetan el equilibrio entre las decisiones antrópicas y sus efectos en la naturaleza. Los efectos sobre el ambiente y la salud humana por el inadecuado manejo y disposición de los RS han llevado a diferentes países a buscar estrategias para contrarrestar dichos impactos: definiendo un marco normativo para controlar la contaminación ambiental, desarrollando políticas que incentiven la reducción en la generación y buscando esquemas institucionales para estimular la reutilización y reciclado de materiales, entre otros. 
En el caso colombiano, se han suscrito convenios internacionales como el Protocolo de Montreal de 1987, el Convenio de Estocolmo de 2001, hasta convenios ambientales para las conferencias internacionales sobre cambio climático. Esta voluntad política por atacar los problemas ambientales, entre, ellos el manejo y disposición de los RS, se ha evidenciado dentro del caso colombiano en la Constitución Política de 1991, la Ley 142 de 1994, así como la política del Manejo Integral de Residuos Sólidos expresada en el CONPES 3874 de 2016. Otras leyes, resoluciones y decretos se han estipulado para reglamentar la prestación del servicio de aseo, establecer las tarifas del mismo y dar las directrices del aprovechamiento y disposición de los residuos generados.

Por lo tanto, considerando que el manejo adecuado de residuos sólidos puede constituirse en un factor determinante para mejorar las condiciones medio ambientales de las ciudades, con repercusiones favorables tanto para el ambiente como para todos los agentes económicos involucrados en esta actividad, en este capítulo se analizarán los determinantes de la generación de residuos sólidos urbanos en Colombia, haciendo uso de herramientas econométricas para estimar aquellas variables que determinen la generación de dichos residuos, y de esta forma proponer alternativas que aporten al diseño de políticas públicas.

\section{La problemática de la Gestión Integral de Residuos Só- lidos en Colombia}

La creciente generación de residuos se encuentra asociada con el aumento demográfico, el crecimiento económico del país y las dinámicas propias de los grandes centros urbanísticos. Aunado a esto, se observa que en Colombia hay "pocos incentivos económicos, normativos y regulatorios para minimizar la generación de residuos sólidos y aumentar los niveles de aprovechamiento y tratamiento de los mismos" (Consejo Nacional de Política Económica y Social -CONPES-, 2016, p. 10); y los procesos de separación en fuente son deficientes. La gestión inadecuada de la creciente generación de residuos sólidos trae como consecuencia problemas de salud por contaminación visual y de aguas residuales. Es así como "el alto consumo de bienes y servicios y el inadecuado manejo de los residuos, ha llevado a que el ciudadano los vea como un problema del cual es urgente deshacerse. Al ser expuestos en lugares públicos durante largos periodos 
de tiempo, genera la proliferación de caninos y roedores ocasionando graves problemas de contaminación visual, del aire, del suelo y de los cuerpos de agua" (Secretaría Distrital de Ambiente, 2014). No obstante, los habitantes solo se preocupan porque le retiren de su vista los desechos, es decir, que la logística de recolección funcione eficientemente, más no por las consecuencias negativas que sobre el ambiente se presentan por la excesiva y/o inadecuada disposición de residuos.

En algunas ciudades se presentan ineficiencias en la recolección, que de acuerdo con Ann (1993), se da por varios factores asociados a las falencias de la política pública, al desconocimiento y la falta de interés de los hogares en la separación de los RSD, la informalidad de los recuperadores de oficio, el poco acompañamiento y reconocimiento a su labor y la falta de responsabilidad social del sector privado que favorezca la separación, recolección y la reutilización. Asimismo, el problema de la disposición final se agudiza, en la medida en que los rellenos sanitarios reducen su vida útil y se desaprovechan residuos que podrían utilizarse como materia prima en diferentes procesos productivos, lo que conlleva tanto a problemas ambientales como de la salud humana.

...el 83\% de los residuos sólidos domiciliarios que se generan van a los rellenos sanitarios y solo el $17 \%$ es recuperado por recicladores para su reincorporación al ciclo productivo (DNP y BM, 2015). Si se continúa con la misma dinámica de generación de residuos, sin adecuadas medidas para mejorar su aprovechamiento o tratamiento, y con patrones de producción y consumo insostenibles, en el año 2030 tendremos emergencias sanitarias en la mayoría de ciudades del país y una alta generación de emisiones de GEl (Consejo Nacional de Política Económica y Social -CONPES-, 2016, p. 31).

Por lo anterior, la necesidad de reducir la cantidad de residuos sólidos generados se hace evidente, para lo cual se debe enfatizar en la educación ambiental y en la participación consiente de los ciudadanos para realizar un consumo responsable y hacer separación en fuente que permita la reutilización y la adecuada disposición de los residuos que no pueden ser reincorporados a la actividad económica. Sin embargo, se percibe una débil capacidad institucional para incentivar a la población a hacer separación en la fuente. Aunque existen medidas educativas y pecuniarias (comparendo ambiental), las personas no realizan este proceso de separación. De acuerdo 
con Novoa (2015) los programas de educación ambiental actualmente aplicados van a tener efectos positivos a largo plazo, pero se requieren, además, modelos de pedagogía para que las autoridades apliquen adecuadamente las instituciones existentes.

En la actualidad se está apostando a un modelo de gestión de residuos sólidos denominado "Economía Circular" el cual "busca que el valor de los productos, los materiales y los recursos se mantenga en la economía durante el mayor tiempo posible, y que se reduzca al mínimo la generación de residuos" (Consejo Nacional de Política Económica y Social -CONPES-, 2016, p. 63). Con este modelo, los efectos negativos de la generación excesiva e inadecuada disposición de los residuos sólidos se reducen, además que favorece el desarrollo de nuevos mercados y la puesta en marcha de tecnologías amigables con el medio ambiente. No obstante, para lograr un modelo de Economía Circular eficiente, se requiere de la existencia de mercados organizados para la comercialización del material potencialmente reutilizable, porque en la actualidad el sector productivo no está incorporando el $100 \%$ de este material en su proceso de fabricación y no hay suficientes avances en ciencia, tecnología e innovación que permita el aprovechamiento de los residuos. Para el caso de la capital, por ejemplo, "la ciencia, tecnología e innovación (CTel) se tienen que conjugar para resolver los problemas reales de la ciudad, entre ellos, la contaminación del río Bogotá y la poca capacidad que tienen los rellenos sanitarios" (Dinero, 2015, p. s.p.), situación que no es ajena al resto de ciudades de Colombia.

\section{Y la normatividad, ¿para qué ha servido?}

De la mano con las recomendaciones internacionales, Colombia ha venido desarrollando desde comienzos de la década de los ochenta la normatividad específica para la gestión de residuos sólidos. Esta regulación ha tenido en cuenta la generación, separación, recolección y disposición de los residuos sólidos, enfatizando en aspectos como la producción verde y las políticas pos consumo. En años recientes se ha hecho énfasis en las actividades de aprovechamiento, en especial, lo expuesto en el Decreto 596 de 2016, con el fin de reducir la cantidad de residuos dispuestos en Rellenos Sanitarios y aumentar los beneficios propuestos en la Economía Circular en materia ambiental. En particular, la Política Nacional para la Gestión Integral de Residuos Sólidos, busca "aportar a la transición de un modelo lineal hacia una 
economía circular donde, haciendo uso de la jerarquía en la gestión de los residuos, se prevenga la generación de residuos y se optimice el uso de los recursos para que los productos permanezcan el mayor tiempo posible en el ciclo económico y se aproveche al máximo su materia prima y potencial energético (Consejo Nacional de Política Económica y Social -CONPES-, 2016, p. 43).

Igualmente, se ha venido trabajando sobre el régimen tarifario con el fin de incluir dentro del cobro los costos de aprovechamiento, disposición e infraestructura que se requieren para la gestión adecuada. Así, en la Resolución 720 de 2015 se establece el régimen de regulación tarifaria al que deben someterse las entidades prestadoras del servicio público de aseo $(73 \%$ en todo el país corresponden a empresas privadas) que atiendan en municipios de más de 5.000 suscriptores en áreas urbanas y la metodología que deben utilizar para el cálculo de las tarifas del servicio público de aseo. En el marco de estas normativas el país ha venido evolucionando en algunos indicadores para el sector. Es así como las tasas de cobertura en recolección han aumentado, de acuerdo con cifras del Departamento Nacional de Planeación aproximadamente tres puntos porcentuales en las áreas urbanas y cuatro en las áreas rurales "las coberturas nacionales de recolección pasaron de un 75,8\% en el año 2005 a $80 \%$ en el año 2014, mientras que las coberturas urbanas pasaron de 94,6\% al 97,8\% en el mismo periodo" (Consejo Nacional de Política Económica y Social -CONPES-, 2016, p. 14).

Adicionalmente, de acuerdo con las cifras presentadas por la Superintendencia de Servicios Públicos (2015) se han reducido los sitios de disposición final ilegales, como los botaderos a cielo abierto en donde se ubican el $4 \%$ de los residuos generados. No obstante, aún persisten dificultades en materia de disposición dada la precariedad tecnológica para el tratamiento de los residuos en disposición, el crecimiento en la generación (se estima que se generan 11,6 millones de toneladas de residuos al año y solo se recicla el 17\%) y la poca en la vida útil de los Rellenos Sanitarios existentes en el país (38\% de los mismos no tienen más de tres años). Aunque la normatividad es clara, persisten algunas dificultades en el sector. Aún no hay una verdadera conciencia ciudadana por la separación en fuente; los porcentajes de aprovechamiento son relativamente bajos si se comparan con países de la región, y europeos como Holanda; sin contar las problemáticas 
asociadas a la inclusión formal y digna por parte de los recicladores en la cadena de valor de los desechos.

Por fortuna la actual Política Nacional para la Gestión de Residuos Sólidos, contempla diferentes estrategias para avanzar hacia la economía circular, es decir, reincorporando los residuos sólidos generados como materia prima dentro de los diferentes procesos de producción, promoviendo la cultura ciudadana para la adecuada separación en fuente, generando un marco institucional más eficiente en el que se incluyen acciones para "mejorar el reporte, monitoreo, verificación y divulgación de la información sectorial para el seguimiento de esta política pública" (Consejo Nacional de Política Económica y Social -CONPES-, 2016, p. 11).

\section{Las cifras de los residuos en Colombia}

El Sistema Único de Información (SUI) reporta que en Bolívar, Bogotá y Valle del Cauca se generaron, en promedio, más de 1.150.000 toneladas de residuos sólidos al mes entre el 2002 y 2009. Para el mismo periodo en departamentos como Antioquia, Caldas y César la producción de desechos correspondió a la mitad de lo registrado por los primeros (532.369, 520.481 y 506.608 ton/promedio/mes, respectivamente). Mientras que en Bogotá un ciudadano produce, en promedio, 25 kilos de residuos sólidos en un mes, en el Valle del Cauca, Meta y Quindío la generación per cápita es de 13, 10 y 9 kilos promedio, respectivamente. Por su parte, en departamentos como Huila y Sucre la producción per cápita de desechos es de 1 kilo al mes (figura 1). 
Figura 1. Residuos Producidos y Recolectados por Departamento en Colombia, 2002-2009

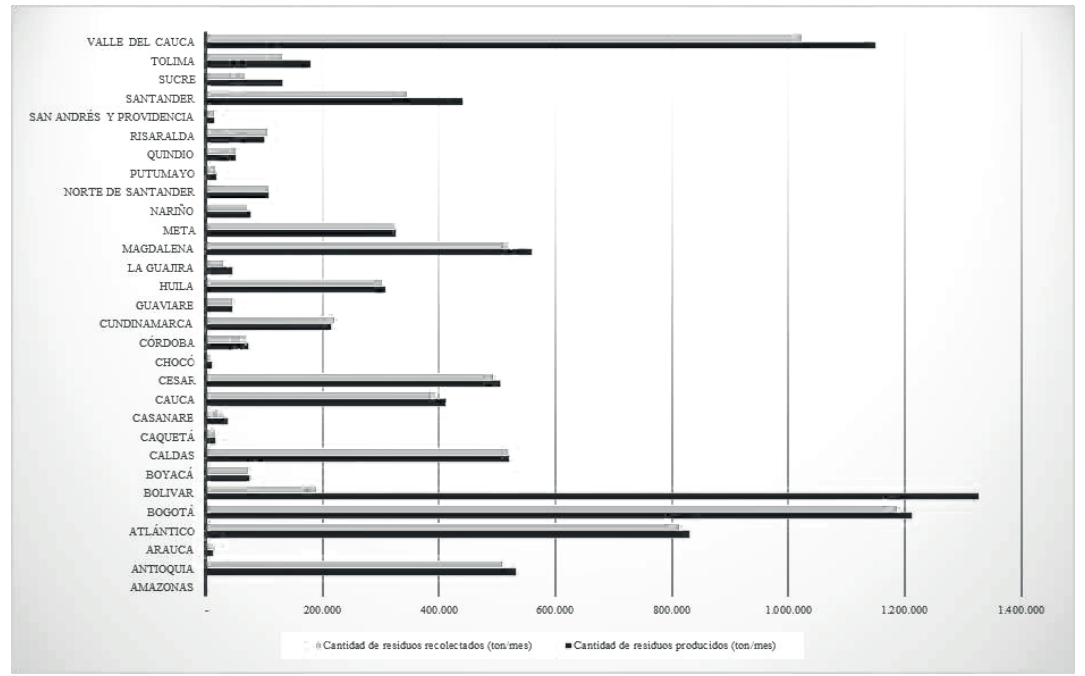

Fuente: Elaboración propia con base en datos del Sistema Único de Información, 2017.

Esto podría estar explicado por las dinámicas particulares de las ciudades y la concentración de las actividades económicas en algunos departamentos (figura 2). En este sentido, se observa una correlación de 0,61 entre el PIB departamental y la producción de residuos sólidos. De acuerdo con la información del SUI, en departamentos con mayor PIB como Antioquia, Bogotá y Valle del Cauca se recolectan más residuos que en otros como Córdoba y Nariño donde, por ejemplo, para el año 2009 el PIB era 6 veces menor.

No se trata solamente de la producción de los residuos sino de la recolección eficiente que se tenga de estos. De acuerdo con el SUI, durante los años 2002 al 2009, 83\% de los residuos producidos fueron en efecto recolectados y llevados a los rellenos sanitarios para su disposición final. Sin embargo, ha de resaltarse que con el paso de los años el porcentaje de residuos efectivamente recolectados respecto al total producido ha venido disminuyendo (tabla 1). 
Figura 2. Correlación entre PIB y Residuos Recolectados por Departamento, 2002-2009

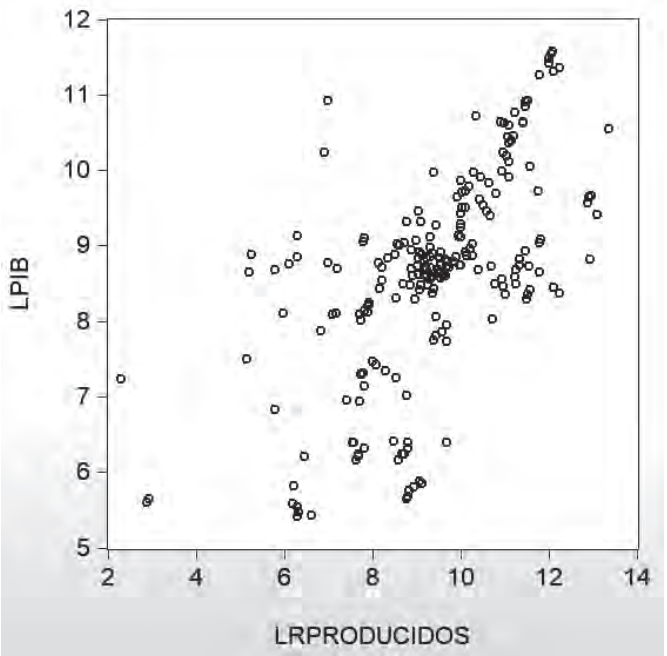

Fuente: Elaboración propia con base en datos del SUI, 2017. Variables departamentales medidas en logaritmo para disminuir errores en las varianzas. Figura elaborada en Eviews.

Tabla 1. Relación entre residuos producidos y residuos recolectados

\begin{tabular}{c|ccc}
\hline Año & Residuos producidos & Residuos recolectados & $\begin{array}{c}\text { Relación Residuos } \\
\text { Recolectados/ } \\
\text { Producidos }\end{array}$ \\
\hline 2002 & 876.048 & 856.802 & $97,80 \%$ \\
\hline 2003 & 1.318 .537 & 1.288 .115 & $97,69 \%$ \\
\hline 2004 & 841.905 & 806.202 & $95,76 \%$ \\
\hline 2005 & 985.980 & 892.324 & $90,50 \%$ \\
\hline 2006 & 2.088 .531 & 1.592 .498 & $76,25 \%$ \\
\hline 2007 & 1.791 .164 & 1.313 .106 & $73,31 \%$ \\
\hline 2008 & 1.340 .845 & 886.124 & $66,09 \%$ \\
\hline 2009 & 99.968 & 58.950 & $58,97 \%$ \\
\hdashline $2002-2009$ & $\mathbf{9 . 3 4 2 . 9 7 8}$ & $\mathbf{7 . 6 9 4 . 1 2 1}$ & $\mathbf{8 2 , 3 5 \%}$ \\
\hline
\end{tabular}

Fuente: Elaboración propia con base en datos del SUI, 2017. Promedio de toneladas por mes. 
Estos resultados implican dos factores importantes: por un lado, la disminución en los residuos recolectados podría estar relacionada con la frecuencia de recolección de los residuos que son finalmente dispuestos en los rellenos sanitarios: en promedio, estos son recolectados 3 veces por semana en zonas residenciales, 4 veces por semana en zonas comerciales y 2 veces por semana en zonas industriales. Por otro lado, existen deficiencias en los registros de información disponibles para el estudio de este tema en Colombia, pues los niveles de incumplimiento con los reportes de residuos producidos y recolectados son elevados y los datos existentes están desactualizados.

Por lo anterior, se busca hacer una aproximación cuantitativa que permita identificar las variables que incrementan la producción de residuos en Colombia, tomando como base las relaciones existentes entre los residuos producidos y el nivel de producción de la economía, junto con la frecuencia de recolección de dichos ${ }^{1}$. Se elaboró un modelo con corrección de errores (VEC, por sus siglas en inglés) para representar el comportamiento dinámico de los residuos producidos en Colombia ${ }^{2}$, y se calculó la ecuación de cointegración que explica la relación simultánea y dependiente de las variables con sus propios rezagos en el tiempo.

Tabla 2. Relaciones de largo plazo en las ecuaciones de integración

\begin{tabular}{l|ccccc} 
& COEFICIENTES DE COINTEGRACIÓN & \\
& LPIB & LResidencial & LComercial & L Industrial & C \\
LRPRODUCIDOS & -1.096818 & 11.69384 & -4.238907 & -2.326795 & \\
& $(0.20875)$ & $(1.95579)$ & $(1.22506)$ & $(0.68554)$ & -4.317014 \\
& {$[-5.25430]$} & {$[5.97909]$} & {$[-3.46017]$} & {$[-3.39411]$} &
\end{tabular}

Fuente: Cálculos propios. Error estándar en ( ), estadístico t en [ ].

Al estimar la ecuación de cointegración se puede concluir, que los niveles de producción de la economía tienen una relación de largo plazo significativa con los niveles de recolección de los residuos sólidos pero inversa dado su coeficiente inferior a 1; es decir, si las

1 Debido a que el modelo está expresado en logaritmo, el resultado implica las elasticidades de los residuos producidos con respecto a las variables consideradas en el estudio. Adicionalmente, los efectos temporales se eliminan antes de la estimación tomando los datos en desviaciones respecto a las medias de corte transversal de cada año.

2 A través del criterio de información de Akaike se estima en dos rezagos la línea de regresión propuesta. 
políticas actualmente adoptadas para mejorar la gestión de residuos sólidos no se consolidan, en el largo plazo se presentará un colapso en la prestación del servicio frente a la cantidad de residuos que se generen por la actividad económica. Igualmente, se destaca la relación con el número de veces que se realizan las recolecciones del sector residencial, implicando que un incremento del 1\% en la frecuencia con la cual se suministra el servicio de recolección de residuos en zonas residenciales se traduciría en un incremento de $11,69 \%$ en la cantidad de residuos recolectados en Colombia en el largo plazo. Esto podría implicar que la mayor frecuencia en recolección desincentiva a los hogares a realizar un consumo responsable y una menor generación de residuos, en la medida en que no tiene que almacenar los desechos por mucho tiempo.

Figura 3. Funciones de respuesta al impulso acumulado

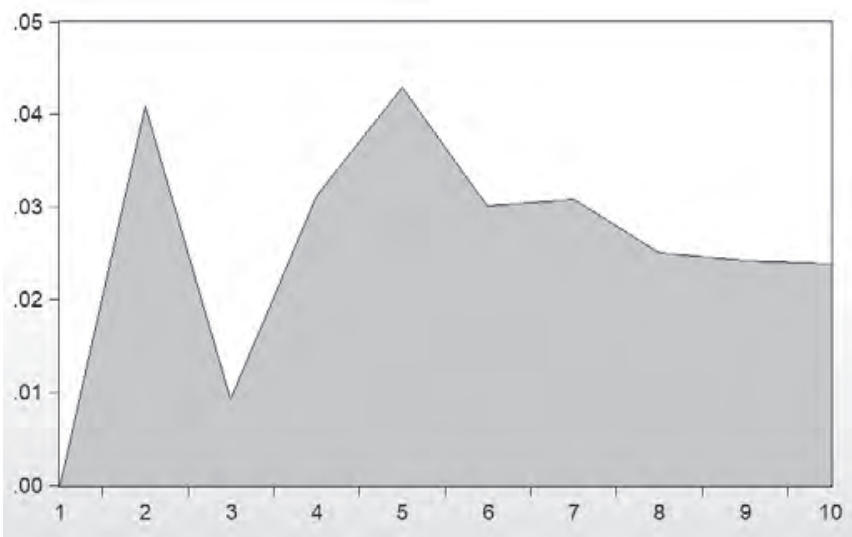

Fuente: Cálculos propios. Gráfico obtenido con el programa Eviews.

Por otro lado, variables como la recolección en zonas comerciales e industriales demuestran un efecto de largo plazo más pequeño, implicando que la generación de residuos en estos sectores no se ve influenciada por la frecuencia en la recolección, sino por las dinámicas propias de sus actividades productivas y de servicios. Adicionalmente, como se evidencia en la figura 3, un impacto de una desviación estándar sobre el PIB de Colombia generaría en el corto plazo un incremento de los residuos sólidos producidos, para posteriormente alcanzar nuevamente un nivel casi constante de producción de residuos. 
Entonces, de acuerdo con el análisis de la información suministrada por la SUI, cerca del $40 \%$ de los residuos producidos no están relacionados con los niveles de producción departamental, por lo que se podría intuir que otros factores pueden tener mayor incidencia en la producción de los mismos. Por ejemplo, que la generación de residuos no depende de decisiones racionales de los individuos ni de niveles de producción de los departamentos, podría estar explicado más por el comportamiento dinámico propio de los hábitos de consumo.

\section{Aportes conclusivos}

Las políticas que buscan reducción en la generación de residuos sólidos deben estar enfocadas en mayor medida a cambiar la conciencia de los hogares, quienes en ultimas, con sus dinámicas de consumo y su poco incentivo para hacer la separación en fuente, se convierten en agentes influyentes para cambiar la tendencia de largo plazo que se está presentando con los desechos. El sector de aseo cuenta con una deficiente base de información que impide hacer un análisis más preciso del mismo. El seguimiento, control y evaluación en la ejecución de la política de manejo de residuos sólidos se dificulta en la medida en que los operadores no hacen el reporte juicioso de sus actividades al Sistema Único de Información (SUI).

Aunque teóricamente se plantea que uno de los determinantes de la generación de residuos sólidos está asociado con las dinámicas de producción de la economía, para el caso Colombiano se evidencia que los desechos generados no son explicados por el comportamiento del PIB. Esto hace inferir que los hábitos de los ciudadanos dentro de la economía consumista y la poca conciencia del adecuado manejo en la fuente de los desechos explican el aumento en la cantidad que se deposita en los diferentes rellenos sanitarios. Si la tendencia continúa a largo plazo, los problemas que afronta este sector se podrían agudizar, por lo que se hace imperante adoptar estrategias para que las instituciones ya creadas no se queden en letra muerta.

Los problemas que se presentan hoy con la vida útil de los rellenos y la falta de capacidad de los mismos para recibir la cantidad de residuos sólidos generados se hubiera podido revertir si las decisiones de política pública no se pospusieran por tantos años. Es increíble 
pensar que después de la promulgación del Decreto 1713 de 2002, el cual presentó los lineamientos para la gestión integral de residuos sólidos, se halla tenido que esperar quince años para tener una política nacional clara de aprovechamiento (Decreto 596 de 2016). Así las cosas, en Colombia no se han dado los incentivos para que los comportamientos de los usuarios del servicio de aseo correspondan a una racionalidad responsable con el medio ambiente.

\section{Bibliografía}

Ann, O. (1993). Los recicladores. Recuperado el 7 de mayo de 2013, de Subgerencia del Banco de la República. Biblioteca Luis Angel Arango: http://www.banrepcultural.org/node/81297

Comisión Mundial sobre Medio Ambiente y el Desarrollo. (1987). (Comisión Brundtland) Nuestro Futuro Común. Oxford, United Reino: Oxford University Press.

Consejo Nacional de Política Económica y Social -CONPES-. (21 de Noviembre de 2016). CONPES 3874: Política Nacional para la Gestión Integral de Residuos Sólidos. 73. Bogotá, Colombia: Departamento Nacional de Planeación.

Dinero. (25 de Agosto de 2015). Las propuestas de los candidatos a la Alcaldía de Bogotá en materia de innovación. Obtenido de http:// www.dinero.com/pais/articulo/alcaldia-bogota-propuestas-materia-ciencia-tecnologia-innovacion/212650

Novoa, J. (26 de Mayo de 2015). A la fecha se han recaudado \$27 millones por comparendos ambientales en Bogotá. (Blu Verde Radio, Entrevistador) Bogotá, Colombia. Obtenido de http://www.bluradio. com/100492/la-fecha-se-han-recaudado-27-millones-por-comparendos-ambientales-en-bogota

Organización Panamericana de la Salud. (2003). Evaluación regional de los servicios de manejo de residuos sólidos municipales: informe analítico de México, evaluación 2002. México D.F.: OPS, Oficina Regional de la Organización Mundial de la Salud. 
Panel Intergubernamental sobre Cambio Climático -IPCC-. (2001). Tercer Informe de Cambio Climático: Las bases científicas. Capítulo 4: Química Atmosférica y Gases Efecto Invernadero. Obtenido de http://www.grida.no/publications/other/ipcc_tar/

Secretaría Distrital de Ambiente. (28 de Noviembre de 2014). Observatorio Ambiental de Bogotá -OAB-. Obtenido de http://oab.ambientebogota.gov.co/es/indicadores?id=184\&v=l

Sen, A. (2001). Desarrollo y Libertad. Bogotá D.C.: Planeta.

Superintendencia de Servicios Públicos Domiciliarios. (2015). Informe Sectorial del Servicio Público de Aseo: grandes prestadores. Bogotá: Superservicios. 